\title{
Ajuda aí, Sinop!: Uma ferramenta para crowdsourcing com gamificação aplicada a cidades inteligentes
}

\author{
Jean Zahn ${ }^{1}$, Mônica da Silva ${ }^{1}$, Erica Mourão ${ }^{1}$, Carlos Gracioli Neto ${ }^{1}$, \\ Erick Simas Grilo ${ }^{1}$, José Viterbo ${ }^{1}$, Flávia Bernardini ${ }^{1}$ \\ ${ }^{1}$ Instituto de Computação - Universidade Federal Fluminense (UFF) \\ Niterói - RJ - Brazil \\ \{jzahn, viterbo, fcbernardini\}eic.uff.br \\ \{monica_silva, ericamourao, carlosgraciolineto, simas_grilo\}eid.uff.br
}

\begin{abstract}
Municipalities continuously present structural problems that demand maintenance. The channels for citizens to report these problems are diverse and in large cities there may be efficient applications. However, the study of the feasibility of using these resources in small and medium-sized cities is essential to support public management. In this study, we prototyping an app aimed at smart cities that uses crowdsourcing and gamification. The prototype was evaluated by usability, satisfaction and technology acceptance methods. The results showed high adoption intent, usefulness and ease of use, compared to traditional forms of interaction between citizens and public authorities.
\end{abstract}

Resumo. Centros urbanos apresentam continuamente problemas estruturais que demandam manutenção. Os canais para o cidadão relatar estes problemas são diversos e em cidades de grande porte podem existir aplicações eficientes. Entretanto, o estudo da viabilidade do uso destes recursos em cidades de pequeno e médio porte é fundamental para o apoio à gestão pública. Neste estudo, realizamos a prototipação de um aplicativo voltado às cidades inteligentes que usa crowdsourcing e gamificação. O protótipo foi avaliado pelos métodos de usabilidade, satisfação e aceitação da tecnologia. Os resultados demonstraram grande intenção de adoção, utilidade e facilidade de uso, em comparação com as formas tradicionais de interação entre o cidadão e o poder público.

\section{Introdução}

Milhares de pessoas transitam em suas cidades e observam situações diversas, assim como problemas relacionados à infraestrutura, saneamento, abastecimento de água, iluminação pública etc. Para resolver esses problemas de forma adequada e rápida, as autoridades públicas responsáveis por esses centros urbanos precisam ter ciência sobre eles. Entretanto, o poder público não tem equipes suficientes para monitorar e acompanhar todas as situações relacionadas a seus municípios [Kandappu et al. 2018]. Neste contexto, o uso de tecnologias digitais permite ampliar a integração entre sociedade e poder público [Kudinov et al. 2019], permitindo ao cidadão realizar reclamações, acompanhamentos, auxiliar em melhorias, e outras atividades em suas cidades.

Em muitas cidades do Brasil, os canais de comunicação existentes entre o cidadão e o órgão público responsáveis por receber solicitação de serviço são: telefone, site da ouvidoria, e-mail, formulários digitais, balcão de atendimento e poucos aplicativos. Com o 
aumento do uso de smartphones e o aumento do acesso à Internet, a criação de aplicativos que permitem a participação ativa de usuários na detecção e resolução desses problemas pode tornar-se mais constante e eficiente para as cidades [Kandappu et al. 2018].

Os cidadãos e o órgão público podem se beneficiar de aplicativos voltados à cidades inteligentes que usam crowdsourcing e gamificação, para registrar e monitorar o status de solicitações [Arakawa and Matsuda 2016]. Porém, existem desafios de gestão, uma vez que os problemas são tratados por departamentos, prestadoras de serviço ou concessionárias que precisam receber as solicitações, apresentar a solução, prover relatórios, etc. Atributos de engajamento como o feedback, apelo estético, sensorial, etc. podem melhorar o processo de relacionamento do cidadão com o setor público [Sampaio et al. 2019], provendo a transparência das informações e conhecimentos sobre a gestão pública através de aplicativos [Kumar 2015]. Aumentar a satisfação e engajamento do cidadão são necessários para manter o uso eficiente desses canais de atendimento [Gooch et al. 2015].

$\mathrm{Na}$ região centro-oeste do Brasil, muitas cidades de pequeno e médio porte ainda utilizam formas pouco sistematizadas de atendimento, por exemplo, e-mail, atendimento pessoal, telefone, etc. Estas cidades enfrentam um crescimento rápido nos últimos anos e com isso a necessidade de melhorias nas formas de atendimento aos cidadãos. A cidade de Sinop, no Estado de Mato Grosso, é um exemplo de cidade que tem um crescimento de aproximadamente $10 \%$ ao ano. Isso faz com que a prestação de serviços públicos apresente problemas em acompanhar o crescimento da cidade e realizar os atendimentos. Tais cidades muitas vezes ainda possuem áreas rurais, vilas e distritos, que demandam atendimento, onde ferramentas tradicionais não atendem ao cidadão.

Considerando o exposto sobre o perfil dos cidadãos do município, identificou-se como questão de pesquisa: é viável o uso de aplicativos de citizen-sourcing para apoio à gestão pública na identificação de problemas em municípios de pequeno e médio porte? Para responder essa questão de pesquisa, elaboramos uma proposta de ferramenta voltada a cidades de pequeno e médio porte, com é o caso de Sinop no Estado de Mato Grosso. Para estimular o engajamento a aplicação foi denominada "Ajuda aí, Sinop!”. A ferramenta visa oferecer à comunidade e aos órgãos de gestão pública uma integração das informações e transparência das ações [Min et al. 2019], garantindo um acompanhamento eficiente, melhor gestão e a aplicação de soluções precisas. Cada cidadão usuário da ferramenta passa a ser um potencial agente fiscalizador, onde a colaboração e a cooperação tornam-se ferramentas no processo. Além disso, existe a possibilidade das pessoas realizarem o relato dos problemas identificados no formato assíncrono, i.e., o cidadão pode estar em uma zona rural ou parte da cidade sem o sinal de internet e após a observar o problema e captura das informações, pode relatar quando obtiver acesso a internet.

Para avaliar a viabilidade do aplicativo, desenvolvemos um protótipo da ferramenta e realizamos um experimento com 18 participantes, que utilizou apresentações on-line, via Google Meet. Comparamos o modelo tradicional, que compreende os procedimentos atualmente utilizados para receber reclamações, sugestões, etc. com o aplicativo proposto baseado em crowdsourcing e gamificação. Após cada etapa da apresentação os participantes responderam a um questionário online. Objetivando um público alvo específico, foram entrevistados cidadãos do município de Sinop, o que possibilitou a realização da avaliação de usabilidade, de satisfação e de aceitação da tecnologia. 
As contribuições deste trabalho abrangem: (i) a realização de um experimento para uma análise comparativa entre o processo atual de reclamação de problemas e a abordagem proposta (via aplicativo); (ii) a avaliação da usabilidade comparativa utilizando o método System Usability Scale (SUS); e (iii) a avaliação da aceitação através do método Technology Acceptance Model (TAM) verificando a facilidade de uso, utilidade e intenção de uso de um aplicativo que promova o uso de dados da multidão (crowdsourcing) e técnicas de gamificação em cidades de pequeno e médio porte.

Este trabalho está organizado da seguinte forma. A Seção 2 descreve a fundamentação teórica. A Seção 3 apresenta a metodologia de pesquisa utilizada para a condução do experimento. A Seção 4 apresenta os resultados e a discussão do cenário apresentado. Por fim, a Seção 5 apresenta as considerações finais.

\section{Fundamentação Teórica}

No contexto urbano, há um amplo discurso da cidade como inteligente, inovadora, digital, criativa, que muitas vezes associa as transformações das Tecnologias da Informação e Comunicação (TIC) com mudanças econômica, política e sociocultural [Bernardini et al. 2020]. A cidade inteligente precisa se basear em algo além do uso de TIC. Ela deve ter uma infraestrutura adequada, apoiar as pessoas e como elas interagem. Além disso, um dos aspectos importante da tecnologia da informação não é necessariamente criar comunidades inteligentes, mas sua adaptabilidade para ser utilizada socialmente em formas que capacitam e educam pessoas, e as envolvem em um debate político sobre suas vidas e o ambiente urbano que habitam [Hollands 2008].

As cidades inteligentes atuam em seis áreas principais e uma delas é a governança, que está relacionada com a interação entre entidades e instituições públicas ou privadas, de forma com que a cidade funcione como organismo vivo [Aires and Santos 2016]. O crowdsourcing é uma abordagem que pode ser utilizada na solução de muitos problemas em uma cidade aliada a governança [Buecheler et al. 2010], e pode ser integrada a coletas de dados gerados por sensores (crowdsensing), para melhorar a obtenção de dados [da Silva et al. 2018]. Especificamente, o uso do crowdsourcing para apoiar a identificação e resolução de problemas públicos é denominado citizenSourcing, e tem potencial para aumentar a participação do cidadão no contexto do governo eletrônico [Monteiro et al. 2021].

O crowdsourcing funciona bem quando os problemas são claramente definidos e as soluções são direcionadas em prazos claros. Ele está relacionado às aplicações massivas onde o progresso deve ser mensurável. Alguns projetos envolvem multidões que relatam fenômenos: qualidade da água, lixo, tráfego, iluminação pública, doações e outros. Os painéis de desempenho do projeto de crowdsourcing e as atualizações regulares servem para melhorar a confiança entre os cidadãos e o estado. Além disso, a parceria entre o governo e a multidão que deseja contribuir com a acumulação de conhecimento e a criação de valor é de extrema importância para a sobrevivência do projeto [Dutil 2015].

Gamificação é um conceito que introduz design e mecanismos de jogos em sistemas para provocar mudanças no comportamento do usuário. A técnica de gamificação pode promover a motivação dos participantes em aplicativos que promovem o uso de dados de multidão (crowd) [Arakawa and Matsuda 2016]. A gamificação no contexto de crowdsourcing pode ser visualizada como uma tentativa de redirecionar a motivação de 
busca de ganho racional para uma determinada atividade intrinsecamente motivada. Elementos de jogos atuam como recursos motivacionais. Podemos citar: pontos, emblemas, tabelas de classificação, avatares e histórias como recursos motivacionais usados com frequência na gamificação [Morschheuser et al. 2017].

\section{Metodologia da Pesquisa}

\subsection{Desenvolvimento da Proposta}

Para a estruturação do experimento foi criado um protótipo, no qual identificamos os requisitos funcionais e não funcionais. A pesquisa envolveu também o estudo de aplicativos similares ao conceito proposto como o Colab.re ${ }^{1}$, ParticipACT ${ }^{2}$ e FixMyStreet ${ }^{3}$. Além disso, identificamos a necessidade da aplicação permitir armazenar e realizar o envio dos dados quando possuir conexão de internet. Em seguida, um protótipo navegável foi desenvolvido na ferramenta de desenvolvimento colaborativo Figma ${ }^{4}$. No protótipo, as contas dos usuários são vinculadas ao Cadastro de Pessoas Físicas (CPF) e aos seus respectivos endereços de e-mail. Para isso, o usuário poderá validar sua identificação através do seu e-mail, com o cadastro o usuário terá acesso a aplicação via tela de login, conforme a Figura 1 (A).
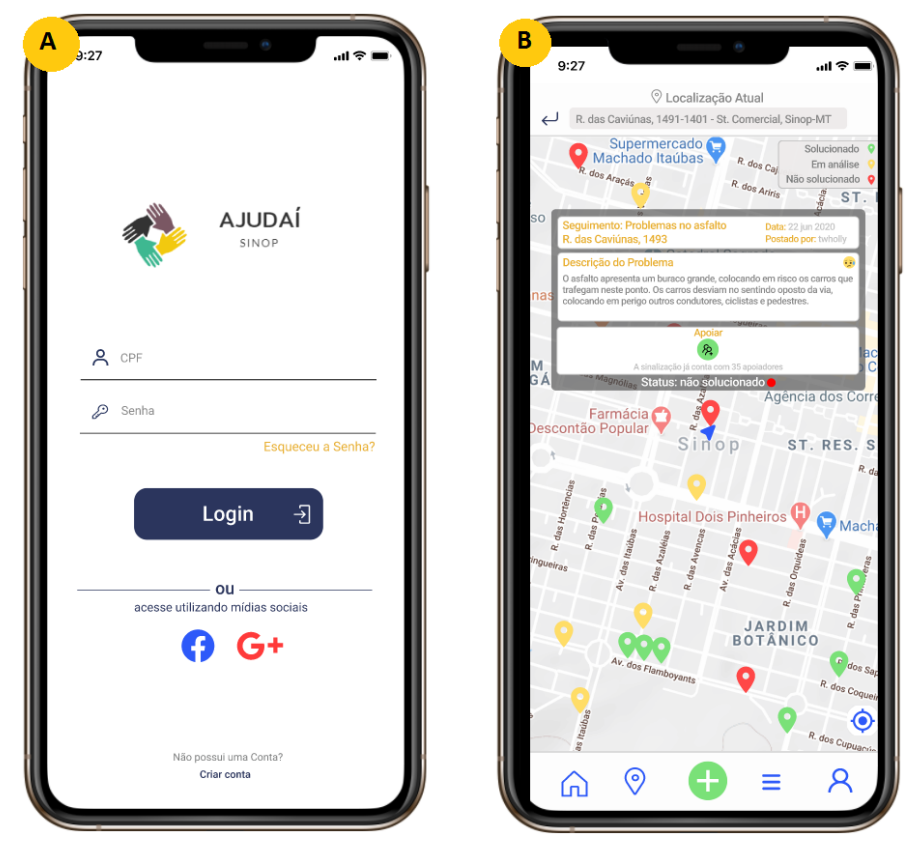

Figura 1. Tela de protótipo - Login (A) e Posicionamento Geográfico (B)

As informações dos usuários são armazenadas na aplicação, mas as informações pessoais, de geolocalização, etc., são protegidas por algoritmos de segurança e processos de anonimização para garantir à privacidade. Essa garantia de privacidade promove maior confiança por parte dos usuário [da Silva et al. 2018], propiciando engajamento no uso da aplicação.

\footnotetext{
${ }^{1}$ https://www.colab.re/

${ }^{2} \mathrm{http}: / /$ www.participact.com.br/

${ }^{3}$ https://www.fixmystreet.com/

${ }^{4}$ https://www.figma.com/
} 
O processo de gamificação aplicado no protótipo utilizou-se de componentes descritos por [Arakawa and Matsuda 2016], que identificou como: tarefa (informar os problemas), recompensa (pontuação maior sendo premiado pelo poder público) e comunicação (a possibilidade de abrir campanhas e compartilhamento em redes sociais).

O uso da aplicação ocorre a partir do acesso do usuário. A posição geográfica atualizada é exibida e o endereço é apresentado na parte superior da ferramenta, conforme observado na Figura 1 (B). Nesta Figura é possível visualizar diversas marcações que representam relatos feitos em lugares próximos ao usuário. Cada marcação apresenta um status: não solucionado (ícone vermelho), em análise (ícone amarelo) ou resolvido (ícone verde).

$\mathrm{Na}$ aplicação o cidadão identifica um problema urbano e deseja sinalizar a gestão pública. Após verificar o endereço, o cidadão poderá escolher o segmento, por exemplo: iluminação pública, esgoto, dano em via pública, etc., e inserir uma descrição do problema. Além disso, fotos podem ser capturadas para contribuir com a identificação do problema. A aplicação também permite armazenar as informações e postar posteriormente. Isso ocorre devido ao fato de existirem localizações sem cobertura de internet, principalmente em áreas rurais.

Por fim, uma campanha poderá ser aberta para dar maior visibilidade ao problema. A possibilidade de habilitar uma campanha com uso de gamificação, foi a maneira encontrada para ganhar caráter de urgência na reclamação. Desse modo, um usuário, em vez de criar uma nova marcação para um mesmo problema, terá a oportunidade de reforçar a marcação que identifica a reclamação criada por outro usuário. Assim, algumas marcações com maior número de apoiadores pode demonstrar maior urgência.

A técnica de gamificação foi inserida no aplicativo como forma de engajar os cidadãos no processo de demarcação dos problemas, que podem ser prioridade. Cada cidadão, ao participar das ações disponíveis no aplicativo, acumula pontos e pode evoluir com o tempo. Os cidadãos mais participativos no aplicativo, ao final de cada ano, podem ser contemplados com premiações ou homenagens do poder legislativo ou executivo do município.

\subsection{Planejamento do Experimento}

Para avaliar a usabilidade e satisfação do modelo tradicional e do aplicativo proposto, foi utilizado o System Usability Scale (SUS) [Brooke 1996]. O método SUS é utilizado para compreender a satisfação e usabilidade durante a interação com os modelos: tradicional e aplicativo proposto. O score do SUS é calculado a partir do resultado de todas as respostas. Para avaliar a aceitação do protótipo do aplicativo proposto foi aplicado o Technology Acceptance Model 3 (TAM 3) [Venkatesh and Bala 2008].

As questões com respostas objetivas irão considerar a Escala Likert [Brooke 1996], que possibilita avaliar as perguntas entre extremos variando por exemplo de: "concordo fortemente" até "discordo fortemente" ou de "concordo"até "discordo", geralmente considerando 05 níveis de concordância. O método SUS contou apenas com questões objetivas baseados na Escala Likert. Entretanto, o TAM utilizou a Escala Likert e também questões discursivas.

$\mathrm{Na}$ execução do experimento foram utilizados 05 (cinco) formulários on-line, 
através da ferramenta Google Forms, sendo: (i) formulário para o termo de consentimento; (ii) formulário para a caracterização dos participantes; (iii) formulário da aplicação do método SUS no modelo tradicional; (iv) formulário da aplicação do método SUS para avaliar o aplicativo proposto; e (v) formulário para avaliar o experimento utilizando o TAM.

Foi criado um cenário contendo dois problemas, simulando a abertura de chamados de problemas de infraestrutura em uma cidade, para ambientar todos os participantes em uma mesma sequência. O primeiro problema compreendia a reclamação de um buraco na via pública, e o segundo problema compreendia a reclamação de vazamento de água potável a empresa companhia de águas da cidade.

\subsection{Realização do Experimento}

Considerando o momento que este estudo foi executado (durante a pandemia de COVID19 em 2020), todas as etapas do experimento foram conduzidas on-line. Para padronizar o fluxo de informações, foram gravados vídeos para cada etapa. Assim todos os participantes receberam a mesma orientação, para evitar influência e diferenças de explicações nos grupos. O primeiro vídeo apresentava as orientações gerais e informações e como as tarefas foram estruturadas. O segundo vídeo compreendia uma apresentação de todas as formas de reclamação que foram encontradas na cidade de Sinop-MT, em cada um dos cenários apresentados anteriormente. O terceiro vídeo apresentou o protótipo da aplicação proposta, descrevendo funcionalidades e possibilidades do uso com gamificação e compartilhamento de campanhas.

O experimento foi planejado pensando em entender a visão geral do público alvo sobre como o processo ocorre atualmente e os benefícios que nossa proposta pode trazer. Para cada problema do cenário, foram executadas duas tarefas conforme o cenário descrito. $\mathrm{O}$ procedimento do experimento foi dividido em três fases:

Preparação ( $1^{\text {a }}$ fase): Um convite foi enviado aos aluno e professores de universidades de Sinop-MT. Os participantes que aceitaram receberam um e-mail com os formulários do Termo de Consentimento e o Questionário de Perfil do Participante.

Execução ( $2^{\mathrm{a}}$ fase): Foram executadas duas rodadas de experimentos, onde a primeira rodada contou com 13 participantes, e a segunda com 05 participantes. Em ambas as rodadas, os participantes executaram as tarefas, mas em ordens invertida. O primeiro grupo executou primeiro a tarefa 1 e em seguida a tarefa 2, enquanto o segundo grupo foi ao contrário, para evitar viés de influenciar sempre os dados na mesma ordem.

Coleta ( $3^{\mathrm{a}}$ fase): Após o experimento, foi realizada a análise dos dados obtidos com o perfil dos participantes, avaliação dos questionários SUS e do formulário TAM.

\section{Discussão dos Resultados}

\subsection{Perfil dos participantes}

Um total de 18 participantes $(\mathrm{P})$ realizaram o experimento, sendo a faixa etária entre 17 e 40 anos. Aproximadamente $61 \%$ dos participantes já efetuaram reclamações de problemas da cidade. Dessas, apenas $14 \%$ utilizaram um meio on-line para efetuar a reclamação e 7\% utilizaram um aplicativo. O restante recorreu a ligações por telefone ou indo pessoalmente aos órgãos responsáveis, somando um total de 50\%. 
Observamos que a faixa etária dos participantes se situa abaixo dos 40 anos. Trabalhos futuros são necessários, uma vez que a população mais idosa tende a achar que "a tecnologia não é feita para o uso deles ou não é relevante" [Van De Watering 2005].

Quanto ao uso dos sistemas colaborativos, um total de $72 \%$ dos participantes declararam nunca ter utilizado um sistema de colaboração e $28 \%$ já utilizaram. Entre os quesitos que os usuários consideraram para decidir utilizar um aplicativo de reclamação voltado à cidade, $89 \%$ participantes consideraram o "nível de satisfação com os municípios por um mecanismo de avaliação" como o tópico mais relevante. Sendo que $78 \%$ consideraram o tempo médio da solução do problema como muito relevante. Por último, $61 \%$ dos participantes acham relevante considerar "os serviços prestados pela gestão pública em um determinado período/região para usar.

\subsection{Avaliação do Método SUS do Aplicativo Proposto}

A métrica SUS foi utilizada para avaliar a usabilidade e satisfação da proposta do aplicativo com 10 questões. Dentre essas, verificamos que ao questionar os participantes se "Gostaria de utilizar este aplicativo frequentemente", 56\% responderam que concordam fortemente, enquanto $39 \%$ responderam que concordam e $6 \%$ são neutros.

Ao avaliar a facilidade de uso, com a afirmação "Achei fácil de utilizar este aplicativo", 44\% participantes que responderam que concordam fortemente, enquanto $44 \%$ responderam que concordam, 6\% se posicionou neutro e 6\% discordaram. Já para a afirmação "Achei este aplicativo complexo", 33\% participantes discordaram fortemente, enquanto $33 \%$ participantes discordaram e $28 \%$ participantes se posicionaram de forma neutra. A frase "Precisaria de ajuda especializada para utilizar este aplicativo" 44\% dos participantes discordaram fortemente, $44 \%$ participantes discordaram e $11 \%$ foram neutros.

Considerando que $89 \%$ dos participantes concordaram que a maioria das pessoas aprenderiam a usar o aplicativo de forma rápida, 94\% discordaram que o aplicativo é incômodo e nenhum participante destacou que necessita de mais informações para utilizar o aplicativo. A execução de tarefas sem dificuldade e com maior facilidade, fluidez e simplicidade [Sampaio et al. 2019], podendo ser considerado um dos fatores de engajamento.

Em termos de segurança de uso, na afirmação "Me senti seguro utilizando este aplicativo" $39 \%$ dos participantes concordam fortemente, $28 \%$ concordam, $28 \%$ foram neutros e apenas $6 \%$ discordaram. Estas respostas indicam que os participantes se sentem seguros no uso do aplicativo.

\subsection{Avaliação do Método SUS do Modelo Tradicional}

A métrica SUS foi utilizada para avaliar a usabilidade e satisfação dos participantes com as metodologias atualmente utilizadas no serviço público da cidade o que chamamos de "modelo tradicional". Entre as 18 respostas obtidas para cada uma das 10 perguntas afirmativas aplicadas, para a afirmação "Gostaria de continuar utilizando o modelo tradicional”, $61 \%$ participantes discordam, enquanto $17 \%$ discorda fortemente, $11 \%$ responderam neutro e $11 \%$ concordam.

Na avaliação da facilidade de uso, a afirmação "Achei fácil de utilizar este modelo tradicional", $39 \%$ participantes que responderam que discordam, enquanto $17 \%$ respon- 
deram que discordam fortemente, $22 \%$ se posicionaram neutro e $17 \%$ concordou e $6 \%$ concordam fortemente. Além disso, para a frase "Achei este modelo tradicional complexo", $33 \%$ participantes responderam que concordam, outros $33 \%$ participantes foram neutros, $11 \%$ concordaram fortemente, $17 \%$ discordaram e $6 \%$ discordaram fortemente.

Para a afirmação "Achei que havia muita inconsistência no modelo tradicional", $56 \%$ dos participantes responderam que concordam, 17\% participantes foram neutros, $11 \%$ responderam que concordam fortemente e $11 \%$ responderam que discorda e apenas $6 \%$ responderam que discordam fortemente.

Em termos de segurança de uso, para a afirmação "Me senti seguro utilizando este modelo tradicional", $33 \%$ das respostas foram neutras, $28 \%$ discordam, $22 \%$ discordam fortemente, $11 \%$ concordam e $6 \%$ concordam fortemente. Além disso, ao avaliar se "Precisaria aprender muitas coisas antes de utilizar este modelo tradicional", 33\% participantes discordaram, os outros $33 \%$ participantes foram neutros, $28 \%$ concordam e $6 \%$ discordaram fortemente. Observa-se neste caso que os participantes sentiram-se menos seguros ao utilizar as formas de reclamação atuais.

\subsection{Análise Comparativa das Avaliações do Método SUS}

Os resultados do uso da metodologia SUS para avaliar usabilidade do aplicativo proposto indica uma maior satisfação, com um score de 81.40, que ficou acima da média desejável de 68 pontos, conforme demonstra a Figura 2. Uma comparação como o resultado do SUS aplicado ao modelo tradicional, que recebeu um score de satisfação de 39.52 pontos. Utilizamos um intervalo de confiança de $95 \%$ e realizamos teste de Wilcoxon pareado para amostras do SUS por participantes, que é usado para identificar qual das amostra apresentou significância estatística [Micallef et al. 2017]. Obtivemos um p-value 0,003 e concluímos que existe diferença significativa para os resultados, isto é, que a satisfação foi maior para a proposta do aplicativo.

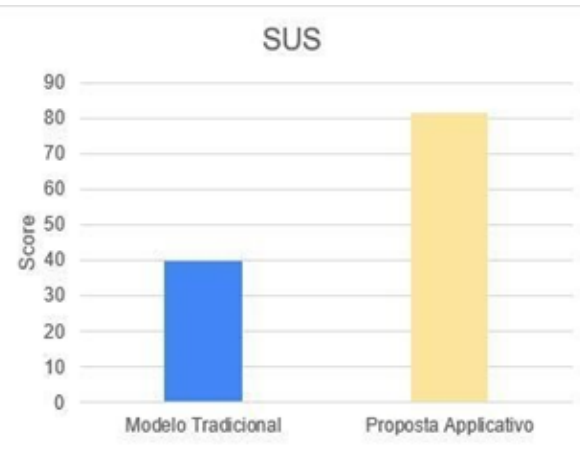

Figura 2. Score SUS: modelo tradicional $\mathrm{X}$ aplicativo proposto

\subsection{Análise da Aceitação do Aplicativo Proposto}

Com base nos dados coletados através do TAM, foi possível avaliar a aceitação dos participantes ao aplicativo proposto em comparação ao modelo tradicional. Também foi possível avaliar a realização do experimento, já esse foi totalmente on-line devido a pandemia de Covid-19. 
Tabela 1. Percepção dos participantes sobre o experimento

\begin{tabular}{|c|c|c|c|c|c|}
\hline Percepção do experimento & Discordo (\%) & $\begin{array}{c}\text { Discordo } \\
\text { Parcialmente }(\%)\end{array}$ & Neutro $(\%)$ & $\begin{array}{c}\text { Concordo } \\
\text { Parcialmente }(\%)\end{array}$ & Concordo $(\%)$ \\
\hline $\begin{array}{l}\text { Eu participaria de outro experimento } \\
\text { on-line }\end{array}$ & 0,0 & 0,0 & 5,6 & 11,1 & 83,3 \\
\hline Foi muito cansativo & 44,4 & 27,8 & 22,2 & 5,6 & 0,0 \\
\hline Me senti a vontade no experimento & 0,0 & 0,0 & 11,1 & 16,7 & 72,2 \\
\hline Consegui me expressar naturalmente & 0,0 & 0,0 & 11,1 & 16,7 & 72,2 \\
\hline Consegui acompanhar o experimento & 0,0 & 0,0 & 11,1 & 11,1 & 77,8 \\
\hline
\end{tabular}

\subsubsection{Percepção do Experimento}

Em relação ao tempo do experimento, todos consideraram ter sido suficiente e, embora globalmente os participantes $(94,4 \%)$ consideraram que o aplicativo não precisasse de orientações adicionais, um participante sugeriu o recebimento de debugs e relatório de versões. Embora globalmente o processo tradicional de abertura de reclamações fosse considerado complexo pelos participantes, os participantes consideraram mais simples o experimento com a utilização do aplicativo. Assim, o aplicativo parece ter reduzido a complexidade percebida. Por exemplo, o participante $\mathrm{P} 2$ mencionou que o processo tradicional de reclamações é "um processo muito tedioso e robusto, tomando muito tempo e sem fácil retorno e nem acessibilidade", enquanto que o participante P8 destacou que o "formato tradicional é um método exaustivo e muitas das vezes ineficaz para solucionar problemas da comunidade de Sinop, visto que a dificuldade para conseguir realizar uma queixa é extremamente alta e nem todas as pessoas contemplam de conhecimento para se localizar em websites mais complexos, reduzindo assim o público alvo". Enquanto que o participante P17 destacou que "o aplicativo é bem intuitivo e está literalmente na mão do usuário".

Devido ao experimento ter sido realizado totalmente on-line, a percepção dos participantes sobre a realização do mesmo pode indicar a viabilidade do processo. Em relação ao experimento, $88,9 \%$ dos participantes nunca participaram de um experimento presencial, sendo que dos 18 participantes $77,8 \%$ nunca haviam participado de um experimento on-line, e todos consideraram uma boa experiência. O participante $\mathrm{P} 3$ afirmou que "tendo em vista que foi o primeiro que participei, foi um bom experimento, foi direto $e$ conciso", enquanto que o participante P17 destacou que "[...] o experimento foi bem aplicado garantindo a integridade da pesquisa”. Todavia, o participante P12 sugeriu alguns ajustes, afirmando que o experimento poderia "melhorar em alguns quesitos, como as apresentações em vídeo tornando-as mais objetivas e profissionais, mas foi um excelente experimento, consegui acompanhar facilmente [...]".

Em geral, os participantes se sentiram à vontade no experimento, percebendo um fácil acompanhamento, conforme Tabela 1. O único ponto a se destacar é que a maioria dos participantes considerou o experimento on-line cansativo e, por isso, sugere-se para os próximos, uma forma de tornar o experimento mais objetivo, conciso e dinâmico.

\subsubsection{Avaliação da Facilidade de Uso}

A facilidade de uso da aplicação proposta foi avaliada na Figura 3 (A), onde 88,8\% dos participantes concordaram com a afirmação "Seria fácil de usar". Um total de 83,2\% dos 
participantes concordaram com a afirmação "Seria mais fácil reclamar". Para a afirmação "Acompanhar as reclamações é fácil" um total de $61,1 \%$ concordaram e 38,9\% concordaram parcialmente.

\subsubsection{Avaliação da Utilidade}

A utilidade do aplicativo foi avaliada na Figura 3 (B), onde 88,9\% dos participantes concordam que o aplicativo "tornaria mais fácil o relato problemas". Além disso, 55,6\% concordam e 33,3\% concordam parcialmente que o aplicativo "tornaria as ações do poder público mais transparentes". Um total de $77,8 \%$ concordam que "melhoraria o desempenho em relatar os problemas". E por último, 83,3\% concordam simultaneamente que o aplicativo "seria útil" e "mais rápido e prático" para relatar problemas e acompanhar.

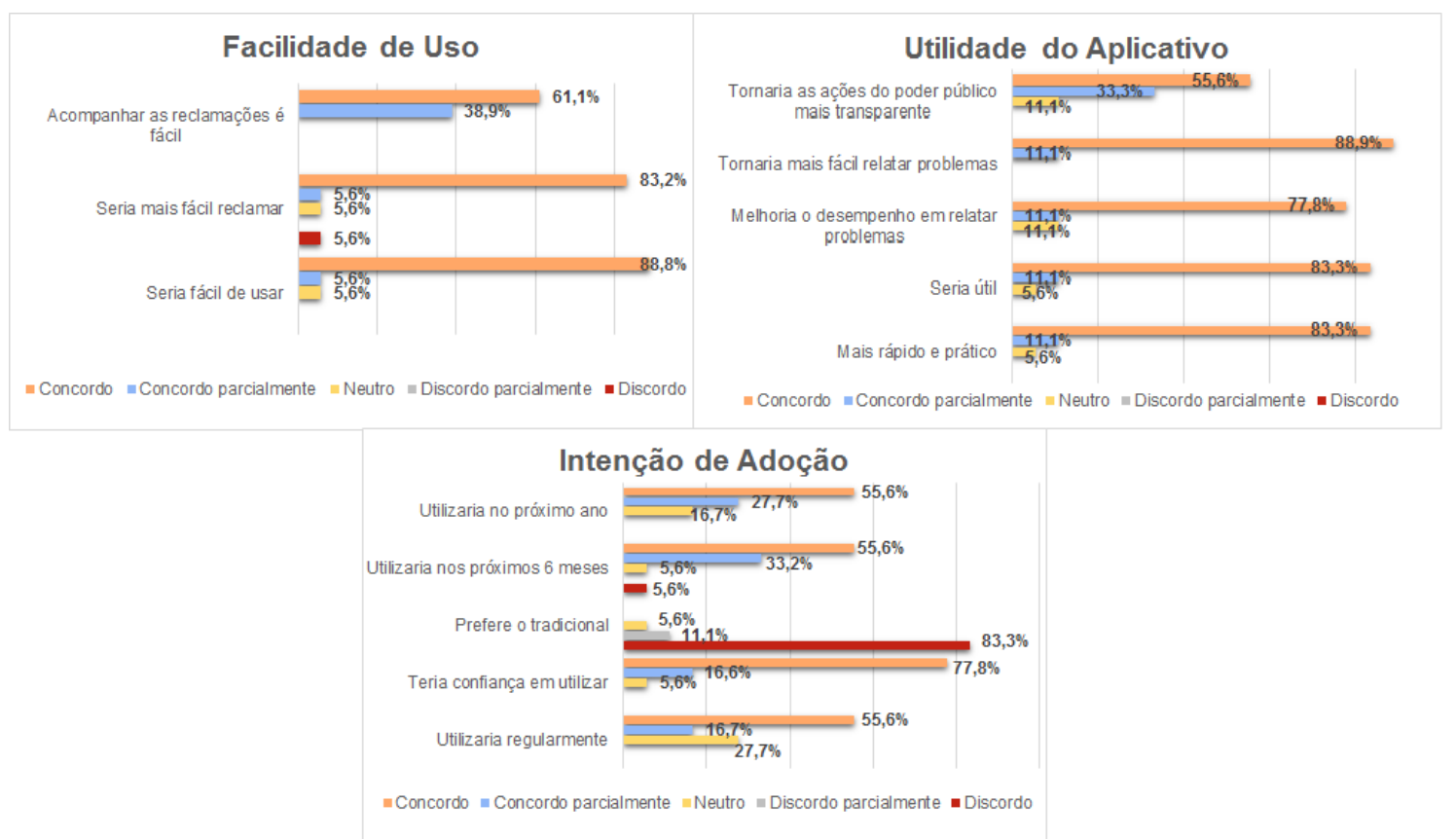

Figura 3. Avaliação TAM: Facilidade de Uso (A); Utilidade do Aplicativo (B); e Intensão de Adoção (C)

\subsubsection{Avaliação da Intenção de Adoção}

Ao avaliar a intenção de adoção ao aplicativo observa-se no gráfico da Figura 3 (C) grande intenção de uso em curto prazo, isto é, em 06 meses e 01 ano onde 55,6\% concordam. Ao mesmo tempo, 77,8 \% dos participantes concordam que "teriam confiança em utilizar" a aplicação. Um total de 55,6\% concordaram que "utilizariam regularmente" o aplicativo. Consequentemente, $83,3 \%$ dos participantes rejeitaram o modelo tradicional.

\section{Considerações Finais}

Problemas de infraestrutura são uma realidade em todas as cidades e demandam de constante acompanhamento e fiscalização por parte da gestão pública. Um cidadão que deseja 
relatar problemas observados na cidade à gestão pública acaba tendo dificuldade em realizar, acompanhar e visualizar a solução do problema, em muitos casos, devido à falta de acesso às informações. A evolução das tecnologias permite que as soluções voltadas a problemas das cidades sejam criadas. O uso de aplicativos com técnicas de inteligência coletiva (crowdsourcing) e gamificação, podem contribuir no desenvolvimento de aplicações que permitam as pessoas interagir para relatar, acompanhar, denunciar problemas e outras situações.

Este trabalho propôs uma abordagem para melhorar o processo de abertura e acompanhamento de problemas em uma cidade de pequeno e médio porte. A proposta foi avaliada em duas rodadas de experimentos, compreendendo um total de 18 participantes, onde foi realizada uma comparação entre o aplicativo proposto e o modelo tradicional para relatar problemas. Os resultados do método SUS apontam que a satisfação no uso do aplicativo foi maior do que o modelo tradicional de abertura de reclamação de problemas na cidade. Em relação aos resultados do método TAM, as percepções da facilidade de uso, utilidade e intenção de adoção demonstram que nossa proposta é mais vantajosa e apresenta viabilidade de uso, comparado ao modelo atual, indicando altos níveis de aprovação. Como trabalhos futuros, pretendemos realizar novos experimentos para avaliar a viabilidade do aplicativo proposto, levando em consideração o perfil de indivíduos idosos e pessoas que residem em áreas rurais de cidades de pequeno e médio porte.

\section{Referências}

Aires, J. and Santos, V. (2016). The use of information tecnologies in the context of smart cities in large cities: The lisbon case study. In 2016 11th Iberian Conference on Information Systems and Technologies (CISTI), pages 1-6. IEEE.

Arakawa, Y. and Matsuda, Y. (2016). Gamification mechanism for enhancing a participatory urban sensing: Survey and practical results. Journal of Information Processing, 24(1):31-38.

Bernardini, F., Viterbo, J., Cappelli, C., and Berger, M. (2020). As cidades do futuro e a computação. In Maciel, C. and Viterbo, J., editors, Computação e Sociedade - Volume 2 - A Sociedade, chapter 11, pages 81-107. EdUFMT, Cuiabá.

Brooke, J. (1996). SUS: a "quick and dirty'usability. Usability evaluation in industry, 189.

Buecheler, T., Sieg, J. H., Füchslin, R. M., and Pfeifer, R. (2010). Crowdsourcing, open innovation and collective intelligence in the scientific method: a research agenda and operational framework. In The 12th International Conference on the Synthesis and Simulation of Living Systems, Odense, Denmark, 19-23 August 2010, pages 679-686. MIT Press.

da Silva, M., Viterbo, J., Bernardini, F., and Maciel, C. (2018). Identifying privacy functional requirements for crowdsourcing applications in smart cities. In 2018 IEEE International Conference on Intelligence and Security Informatics (ISI), pages 106-111. IEEE.

Dutil, P. (2015). Crowdsourcing as a new instrument in the government's arsenal: Explorations and considerations. Canadian Public Administration, 58(3):363-383. 
Gooch, D., Wolff, A., Kortuem, G., and Brown, R. (2015). Reimagining the role of citizens in smart city projects. In Adjunct Proceedings of the 2015 ACM International Joint Conference on Pervasive and Ubiquitous Computing and Proceedings of the 2015 ACM International Symposium on Wearable Computers, pages 1587-1594.

Hollands, R. G. (2008). Will the real smart city please stand up? intelligent, progressive or entrepreneurial? City, 12(3):303-320.

Kandappu, T., Misra, A., Koh, D., Tandriansyah, R. D., and Jaiman, N. (2018). A feasibility study on crowdsourcing to monitor municipal resources in smart cities. In Companion Proceedings of the The Web Conference 2018, pages 919-925.

Kudinov, S., Ilina, E., and Antonov, A. (2019). Analyzing civic activity in the field of urban improvement and housing maintenance based on e-participation data: St. petersburg experience. In International Conference on Electronic Governance and Open Society: Challenges in Eurasia, pages 88-102. Springer.

Kumar, T. V. (2015). E-governance for smart cities. In E-governance for smart cities, pages $1-43$. Springer.

Micallef, N., Just, M., Baillie, L., and Alharby, M. (2017). Stop annoying me! an empirical investigation of the usability of app privacy notifications. In Proceedings of the 29th Australian Conference on Computer-Human Interaction, pages 371-375.

Min, K., Jun, B., Lee, J., Kim, H., and Furuya, K. (2019). Analysis of environmental issues with an application of civil complaints: The case of shiheung city, republic of korea. International journal of environmental research and public health, 16(6):1018.

Monteiro, M., Vasconcelos, L., Viterbo, J., Salgado, L., and Bernardini, F. (2021). Assessing the quality of local e-government service through citizen-sourcing applications. In 2021 IEEE 24th International Conference on Computer Supported Cooperative Work in Design (CSCWD), pages 1178-1183.

Morschheuser, B., Hamari, J., Koivisto, J., and Maedche, A. (2017). Gamified crowdsourcing: Conceptualization, literature review, and future agenda. International Journal of Human-Computer Studies, 106:26-43.

Sampaio, I., Vasconcelos, L., Viterbo, J., and Trevisan, D. (2019). Percepção do usuário quanto aos atributos de engajamento: uma análise qualitativa. In Anais do X Workshop sobre Aspectos da Interação Humano-Computador para a Web Social, pages 51-60, Porto Alegre, RS, Brasil. SBC.

Van De Watering, M. (2005). The impact of computer technology on the elderly. Retrieved June, 29(2008):12.

Venkatesh, V. and Bala, H. (2008). Technology acceptance model 3 and a research agenda on interventions. Decision sciences, 39(2):273-315. 